\title{
Correlação entre o aumento do volume orbitário pós trauma em face e as repercussões clínicas
}

\author{
Correlation between increasing the post-trauma orbitary volume and as clinical repercussions \\ Correlación entre el incremento del volumen orbitario post-trauma y las repercusiones clínicas
}

Recebido: 05/05/2021 | Revisado: 12/05/2021 | Aceito: 18/05/2021 | Publicado: 04/06/2021

Lucas Emmanuel de Moraes Neves

ORCID: https://orcid.org/0000-0001-7257-3148 Hospital da Restauração, Brasil

E-mail: lucas_emmanuell@hotmail.com

Maxsuel Bezerra da Silva

ORCID: https://orcid.org/0000-0003-4366-9508 Hospital da Restauração, Brasil

E-mail: maxsuelmd@ hotmail.com

Felipe Ricardo Cisneiros Brito

ORCID: https://orcid.org/0000-0001-8222-9033

Faculdade de Odontologia de Pernambuco, Brasil E-mail: felipe.cisneiros@upe.br

Greiciane Miguel de Azevedo Santos

ORCID: https://orcid.org/0000-0001-7876-3655

Faculdade de Odontologia de Pernambuco, Brasil

E-mail: greiciane_azevedo@ outlook.com

Fernanda Kelly Costa Tito

ORCID: https://orcid.org/0000-0001-7481-2587

Universidade Estadual da Paraíba, Brasil

E-mail: ffernandacosttaa@gmail.com

Myllenna dos Santos Ferreira

ORCID: https://orcid.org/0000-0002-4026-0996

Universidade Estadual da Paraíba, Brasil

E-mail: myllenna.aju@gmail.com

Luiza Fernanda Correia Molina Cabral

ORCID: https://orcid.org/0000-0003-2006-0353

Faculdade de Odontologia de Pernambuco, Brasil E-mail: luiza.correia@upe.br

Heverson Thiago da Silva Souza

ORCID: https://orcid.org/0000-0001-5852-4905

Universidade Federal de Pernambuco, Brasil

E-mail: heverson.thiago@ufpe.br

Lilian Juliana Torres Silva

ORCID: https://orcid.org/0000-0002-6753-6219 Universidade Estadual da Paraíba, Brasil

E-mail: lilianjuliana8@gmail.com

Rafaelle Leal de Melo Rocha

ORCID: https://orcid.org/0000-0001-5446-1281 Universidade Estadual da Paraíba, Brasil

E-mail: rafaellelealmelorocha17@gmail.com

\section{Resumo}

As fraturas orbitárias transcorrem com considerável frequência e quando há o envolvimento das suas paredes, geralmente observam-se mudanças no volume orbitário, que resultam em diversas alterações clínicas. Objetiva-se por meio desse estudo, correlacionar o aumento do volume orbitário, pós-trauma em face, com as alterações clínicas observadas, enfatizando a influência dessas variáveis na tomada de decisão sobre a intervenção terapêutica mais adequada. Realizou-se um levantamento bibliográfico nas principais bases de dados online, por meio dos descritores: Órbita, Fraturas Ósseas, Tomografia e Cirurgia Maxilofacial, contemplando artigos completos de pesquisa, revisão, e ainda, relatos de caso, publicados nos últimos 5 anos (2015-2020), nos idiomas inglês, português e espanhol. Por meio do estudo, tornou-se claro que as fraturas orbitais representam $40 \%$ de todas as fraturas faciais, podendo esse percentual aumentar quando há o envolvimento de outros ossos articulados. Evidenciou-se a existência de uma correlação positiva entre o aumento de volume orbitário e a ocorrência de algumas alterações clínicas. Com relação a escolha do tratamento apropriado para a correção das deformidades, compreendeu-se que características clínicas e radiográficas devem ser observadas, sendo a diplopia, distopia, enoftalmia, exolftalmia, oftalmoplegia e lesão nervosa 
as principais alterações clínicas. Dessa maneira, conclui-se que o aumento volumétrico influencia diretamente as repercussões clínicas pós-traumas em face, devendo ser considerado no processo decisões terapêuticas, que podem ser cirúrgicas ou conservadoras.

Palavras-chave: Órbita; Fraturas ósseas; Tomografia; Cirurgia maxilofacial.

\begin{abstract}
Orbital fractures occur with considerable frequency and when there is involvement of its walls, changes in orbital volume are usually observed, resulting in several clinical changes. The objective of this study is to correlate the increase in orbital volume, post-trauma in the face, with the clinical changes observed, emphasizing the influence of these variables on decision making regarding the most appropriate therapeutic intervention. A bibliographic survey was carried out in the main online databases, using the descriptors: Orbit, Bone Fractures, Tomography and Maxillofacial Surgery, covering complete articles of research, review, and also, case reports, published in the last few 5 years (2015-2020), in English, Portuguese and Spanish. Through the study, it became clear that orbital fractures represent $40 \%$ of all facial fractures, and this percentage may increase when other articulated bones are involved. There was a positive correlation between the increase in orbital volume and the occurrence of some clinical changes. Regarding the choice of the appropriate treatment for the correction of deformities, it was understood that clinical and radiographic characteristics must be observed, with diplopia, dystopia, enophthalmia, exolftalmia, ophthalmoplegia and nerve injury being the main clinical changes. In this way, it is concluded that the volumetric increase directly influences the clinical repercussions after trauma in the face, and therapeutic decisions should be considered in the process, which can be surgical or conservative.
\end{abstract}

Keywords: Orbit; Bone fractures; Tomography; Maxilofacial surgery.

\title{
Resumen
}

Las fracturas orbitarias ocurren con frecuencia considerable y cuando hay compromiso de sus paredes, generalmente se observan cambios en el volumen orbital, lo que resulta en varios cambios clínicos. El objetivo de este estudio es correlacionar el aumento del volumen orbitario, postraumático en la cara, con los cambios clínicos observados, enfatizando la influencia de estas variables en la toma de decisiones con respecto a la intervención terapéutica más adecuada. Se realizó una encuesta bibliográfica en las principales bases de datos en línea, utilizando los descriptores: órbita, fracturas óseas, tomografía y cirugía maxilofacial, que abarca artículos completos de investigación, revisión y también informes de casos publicados en los últimos 5 años (2015-2020), en inglés, portugués y español. A través del estudio, quedó claro que las fracturas orbitales representan el $40 \%$ de todas las fracturas faciales, y este porcentaje puede aumentar cuando están involucrados otros huesos articulados. Hubo una correlación positiva entre el aumento del volumen orbital y la aparición de algunos cambios clínicos. En cuanto a la elección del tratamiento apropiado para la corrección de las deformidades, se entendió que las características clínicas y radiográficas deben observarse, siendo la diplopía, la distopía, la enoftalmia, la exolftalmia, la oftalmoplejía y la lesión nerviosa los principales cambios clínicos. De esta manera, se concluye que el aumento volumétrico influye directamente en las repercusiones clínicas después del trauma en la cara, y las decisiones terapéuticas deben considerarse en el proceso, que puede ser quirúrgico o conservador.

Palabras clave: Órbita; Fracturas ósseas; Tomografía; Cirugía maxilofacial.

\section{Introdução}

Devido a fatores como exposição e mínima proteção da área de impacto, a face torna-se mais suscetível a ocorrência de traumas, sendo frequentemente encontrados indivíduos cursando com fraturas ósseas nessa região em hospitais com serviços de urgência e emergência (Lozada et al., 2019).

Entre os diversos tipos de trauma em face, as fraturas orbitárias transcorrem com considerável frequência, representando aproximadamente $40 \%$ de todas as fraturas faciais (Oliveira et al., 2019). A morfofisiologia da face é bastante complexa e suas implicações no trauma geram comportamentos diferentes em cada região afetada (Andrades et al., 2018).

Estudos epidemiológicos descreveram que $75 \%$ dos pacientes do sexo masculino, com idade média variando entre 15 a 40 anos e história de trauma de alta energia, encontram-se mais suscetíveis à ocorrência de fraturas em paredes de órbita (Borad et al., 2017). Nesse contexto, o estabelecimento de um diagnóstico rápido, minucioso e eficaz, faz-se imprescindível no manejo de traumas orbitários, possibilitando a elaboração de um plano de tratamento adequado e consequentemente a escolha de uma abordagem terapêutica apropriada.

As fraturas orbitárias podem ocorrer isoladamente ou com envolvimento de outros ossos da face. Quando há envolvimento das paredes orbitárias, comumente se observa uma mudança no volume orbitário, resultando em diversas 
alterações clínicas, tais como edema e equimose periorbitários, hemorragia subconjuntival, diplopia, distopia, enoftalmia e exolftalmia, oftalmoplegia, lesão nervosa, perfuração do globo ocular e ferimentos em tecidos moles (El-Mahallawy \& AlMahalawy 2020).

Os exames de imagem são ferramentas extremamente úteis no processo de diagnóstico por fornecerem informações complementares ao exame físico (Oliveira et al., 2019). A tomografia computadorizada (TC) representa uma importante aliada no manejo das fraturas de órbita, uma vez que por meio dela, as alterações no volume orbitário podem ser facilmente medidas, proporcionando o estabelecimento de um planejamento pré-operatório e avaliação pós-operatória mais adequada (Nilsson et al., 2018).

A correlação entre o aumento do volume da órbita e o desenvolvimento de alterações clínicas vem sendo abordada nos estudos e discussões da literatura atual, os quais têm sugerido o uso dessa associação para obtenção de previsibilidade das evoluções clínicas após alteração no volume orbitário, influenciando deliberações a respeito da escolha do tratamento, podendo ser este, conservador ou cirúrgico (El-Mahallawy \& Al-Mahalawy 2020).

O manejo das fraturas orbitais requer bastante cautela e um planejamento multidisciplinar entre as equipes de cirurgia e traumatologia buco-maxilo-facial, oftalmologia e neurocirurgia. Contudo, a seleção do tratamento seja ele conservador ou cirúrgico, para correção de deformidades, deve levar em consideração características clínicas e radiográficas, as quais devem ser confrontadas, subsidiando uma conduta adequada (Marano et al., 2019).

O tratamento cirúrgico para as fraturas orbitais visa restaurar a anatomia óssea por meio da correção do posicionamento do globo ocular dentro da cavidade orbitária, auxiliando consequentemente na resolução das alterações clínicas desenvolvidas pelo paciente (Byeon \& Choi 2017; Nilsson et al., 2018; Oliveira et al., 2019).

Ante o exposto e considerando o limitado número de publicações científicas relevantes abordando o volume orbitário após traumas faciais, o objetivo deste estudo concentra-se em correlacionar a alteração do volume da órbita, decorrente de um trauma facial, com as repercussões clínicas do mesmo, reforçando a influência dessa associação na escolha da intervenção terapêutica mais apropriada.

\section{Metodologia}

Para realização deste estudo de revisão, foram selecionados artigos de pesquisa, relatos de casos e revisões, publicados nos idiomas inglês, português e espanhol nos últimos 5 anos, nos quais as alterações clínicas e volume orbitário, após fratura de parede de órbita, fossem o evento de interesse. As bases de dados empregadas para o rastreamento foram LILACS, PUBMED/MEDLINE e SCIELO. Foram considerados relevantes os estudos que apresentavam levantamentos epidemiológicos, aspectos clínicos e abordagens terapêuticas para fraturas de órbita, objetivando reabilitação estética e funcional. Os estudos foram analisados quanto ao ano de publicação, local de realização, população do estudo, objetivos, metodologias e resultados. Durante um primeiro rastreamento foram incluídos artigos que apresentavam títulos pertinentes ao tema. Posteriormente, foram selecionados resumos das publicações relacionadas a anatomia do volume orbitário e trauma em face. Por fim, os artigos selecionados foram avaliados na íntegra, contabilizando 30 artigos que se enquadravam com informações da temática estabelecida.

\section{Resultados e Discussão}

As fraturas orbitais podem resultar no desenvolvimento de múltiplas afecções oculares. Devido a complexa anatomia da região e ao frequente acometimento do globo ocular, fraturas de paredes da órbita, podem apresentar diversas complicações, com alta morbidade, dentre as quais destaca-se a redução do nível de consciência do paciente (Felding et al., 2016; Oliveira et al., 2019). 
As fraturas de órbita podem apresentar padrões isolados ou envolver outras estruturas ósseas e de tecido mole, como por exemplo, as fraturas do complexo zigomático-maxilar e as fraturas naso-órbito-etmoidais (Rajkumar et al., 2015; Hartwig et al., 2019). A correlação entre o aumento do volume da órbita, verificado por meio de exames complementares, e o desenvolvimento de alterações clínicas tem sido frequentemente discutida na literatura atual (Schönegg et al., 2018; Snäll et al., 2019). Alhamdani et al. (2015) descrevem as principais injúrias oftalmológicas após trauma em face cursando com fraturas em parede de órbita, são elas: hifema, midríase traumática, abrasão da córnea e equimose conjuntival. Allisson et al. (2020) menciona em seu estudo, alteração na posição do globo ocular, baixa acuidade visual, hemorragia subconjuntival e alteração da sensibilidade da divisão maxilar do nervo trigêmeo, como os sinais/sintomas mais específicos das fraturas de paredes de órbita.

De acordo com Roselló et al. (2020), a tomografia computadorizada tridimensional (TC) é um exame complementar e um recurso extremamente útil para a realização de um planejamento pré-operatório e avaliação pós-operatória corretos, permitindo a realização de medições do volume orbital resultantes das fraturas faciais. Corroborando tal estudo, Ramphul, Hoffman (2017) descrevem que a TC de face fornece imagens que evidenciam a disposição e a morfologia da musculatura ocular, sendo bastante útil no manejo das fraturas, proporcionando um diagnóstico rápido e eficaz.

As fraturas do assoalho orbitário apresentam com maior frequência diplopia e encarceramento muscular do músculo reto inferior. Já as fraturas que envolvem a parede medial da órbita, configuram-se como mais desafiadoras para a obtenção do diagnóstico clínico, uma vez que suas repercussões podem ser imperceptíveis ao exame físico, requerendo a realização de exames de imagem que, frequentemente, evidenciam apenas repercussões volumétricas (Shah et al., 2018; Lozada et al., 2019).

Após fraturas em paredes da órbita pode ser observado um aumento do volume orbital, ocasionando diversas alterações clínicas. Alhamdani et al. (2015) descrevem a diplopia como a repercussão clínica mais frequente após trauma em órbita. Andrades et al. (2018) após análises das alterações clínicas e abordagens terapêuticas, relatam que distopia de mais de 02 milímetros pode ser observada clinicamente, auxiliando na decisão da cirurgia.

Uma correlação positiva vem sendo encontrada em muitos estudos, entre o volume orbitário e o grau de enoftalmia. Andrades et al. (2018) em seu estudo, cujo objetivo foi analisar o volume orbitário e a relação clínica no processo de tomada de decisão, descreveram que para cada aumento de 01 cc no volume orbitário, há aproximadamente 01 milímetro de enoftalmia.

Com relação ao posicionamento do globo ocular, Goggin et al. (2015) após análises prévias observaram que traumas com defeito ósseo de $0,6 \mathrm{~cm}^{2}$ resultam em um aumento de $01 \mathrm{~cm}^{3}$ no volume orbital e, consequentemente, ocasionam o deslocamento posterior de 01 milímetro do globo ocular.

O principal objetivo da abordagem terapêutica para fratura de órbita é a exploração e liberação do tecido mole herniado, bem como a restauração do volume orbitário original (Wi et al., 2017). Os procedimentos cirúrgicos quando bem executados resultam em correção da posição do globo dentro da cavidade orbital e auxiliam na correção dos sinais e sintomas visuais (Scolozzi et al., 2017; Nilsson et al., 2018; Oliveira et al., 2019).

Devido às diferentes abordagens para fraturas orbitárias descritas na literatura, ainda há muitas controvérsias no que diz respeito a seleção de um tratamento cirúrgico ou conservador. A interpretação dos achados e a provável resolubilidade espontânea faz com que muitos casos sejam gerenciados de forma conservadora, o que apresenta consideráveis taxas de sucesso (Alhamdani et al., 2015; Jung et al., 2017; Young et al., 2018).

De acordo com Kovar et al. (2018), para defeitos do assoalho orbital, dos quais se espera enoftalmia considerável ou diplopia não decrescente, o tratamento cirúrgico está indicado. Assim, respalda o preconizado por Ebrahimi et al. (2019), que descreveram que a oftalmoplegia por encarceramento de músculos e enoftalmia superior a 3 milímetros são repercussões clínicas relevantes e significativas que indicam o tratamento cirúrgico, exceto em casos de riscos agudos ao globo ocular. 


\section{Conclusão}

Ante o exposto, torna-se clara a importância da associação entre os achados clínicos e as medidas do volume orbitário, permitindo o estabelecimento de um adequado diagnóstico clínico, repercutindo na seleção de uma abordagem terapêutica apropriada. Pode-se ainda inferir que a interpretação correta dos achados volumétricos por meio dos exames complementares tem fundamental importância para a prática do cirurgião bucomaxilofacial, fornecendo informações precisas do trauma e das suas consequências imediatas e/ou tardias.

\section{Referências}

Alhamdani, F., Durham, J., Greenwood, M., \& Corbett, I. (2015). Diplopia and ocular motility in orbital blow-out fractures: 10-year retrospective study. Journal of Cranio-Maxillofacial Surgery, 43(7), 1010-1016.

Allison, J. R., Kearns, A., \& Banks, R. J. (2020). Predicting orbital fractures in head injury: a preliminary study of clinical findings. Emergency radiology, 27(1), 31-36.

Andrades, P., Cuevas, P., Hernández, R., Danilla, S., \& Villalobos, R. (2018). Characterization of the orbital volume in normal population. Journal of CranioMaxillofacial Surgery, 46(4), 594-599.

Borad, V., Lacey, M. S., Hamlar, D. D., Dresner, H. S., Yadava, G. K., \& Schubert, W. (2017). Intraoperative imaging changes management in orbital fracture repair. Journal of Oral and Maxillofacial Surgery, 75(9), 1932-1940.

Byeon, J. Y., \& Choi, H. J. (2017). Orbital cellulitis following orbital blow-out fracture. Journal of Craniofacial Surgery, $28(7), 1777-1779$.

de Oliveira, P. G., da Câmara, C. P., \& Coelho, P. V. (2019). Intra-and interreader variability of orbital volume quantification using 3D computed tomography for reconstructed orbital fractures. Journal of Cranio-Maxillofacial Surgery, 47(7), 1060-1064.

Ebrahimi, A., Motamedi, M. H. K., Rasouli, H. R., \& Naghdi, N. (2019). Enophthalmos and orbital volume changes in zygomaticomaxillary complex fractures: is there a correlation between them?. Journal of Oral and Maxillofacial Surgery, 77(1), $134-\mathrm{e} 1$.

El-Mahallawy, Y. A., \& Al-Mahalawy, H. A. (2020). Evaluation of orbital volume after orbitozygomatic complex fractures fixation: A radiographical study. Journal of oral biology and craniofacial research, 10(2), 66-71.

Felding, U. A., Rasmussen, J., Toft, P. B., \& von Buchwald, C. (2016). The functional outcome of blow-out fractures managed surgically and conservatively: our experience in 100 patients. European Archives of Oto-Rhino-Laryngology, 273(7), 1927-1931.

Goggin, J., Jupiter, D. C., \& Czerwinski, M. (2015). Simple Computed Tomography-Based Calculations of Orbital Floor Fracture Defect Size Are Not Sufficiently Accurate for Clinical Use. Journal of Oral and Maxillofacial Surgery, 73(1), 112-116.

Hartwig, S., Nissen, M. C., Voss, J. O., Doll, C., Adolphs, N., Heiland, M., \& Raguse, J. D. (2019). Clinical outcome after orbital floor fracture reduction with special regard to patient's satisfaction. Chinese Journal of Traumatology, 22(3), 155-160.

Jung, S., Lee, J. W., Kim, C. H., Hwang, E., Lim, H., Jung, S. W., \& Koh, S. H. (2017). Postoperative changes in isolated medial orbital wall fractures based on computed tomography. Journal of Craniofacial Surgery, 28(8), 2038-2041.

Kovar, D., Holy, R., Voldrich, Z., Voska, P., Lestak, J., \& Astl, J. (2018). The application of volumometry as an indication criterion in blow-out fractures. Biomedical Papers, 162(1), 61-64.

Lozada, K. N., Cleveland, P. W., \& Smith, J. E. (2019). Orbital Trauma. Seminars in plastic surgery, 33(2), 106-113. https://doi.org/10.1055/s-0039-1685477

Marano, R., Lino, P. R. S., Zanetti, F., Tincani, A. J., \& Oliveira, L. (2019). Is specialized ophthalmologic evaluation necessary after orbital fractures? A prospective 64-case study. Oral and maxillofacial surgery, 23(3), 325-329.

Nilsson, J., Nysjö, J., Carlsson, A. P., \& Thor, A. (2018). Comparison analysis of orbital shape and volume in unilateral fractured orbits. Journal of CranioMaxillofacial Surgery, 46(3), 381-387.

Rajkumar, G. C., Ashwin, D. P., Singh, R., Prashanth, R., \& Rudresh, K. B. (2015). Ocular injuries associated with midface fractures: A 5 year survey. Journal of maxillofacial and oral surgery, 14(4), 925-929.

Ramphul, A., \& Hoffman, G. (2017). Does preoperative diplopia determine the incidence of postoperative diplopia after repair of orbital floor fracture? An institutional review. Journal of Oral and Maxillofacial Surgery, 75(3), 565-575.

Roselló, E. G., Granado, A. M. Q., Garcia, M. A., Martí, S. J., Sala, G. L., Mármol, B. B., \& Gutiérrez, S. P. (2020). Facial fractures: classification and highlights for a useful report. Insights into imaging, 11(1), 1-15.

Schönegg, D., Wagner, M., Schumann, P., Essig, H., Seifert, B., Rücker, M., \& Gander, T. (2018). Correlation between increased orbital volume and enophthalmos and diplopia in patients with fractures of the orbital floor or the medial orbital wall. Journal of Cranio-Maxillofacial Surgery, 46(9), 1544-1549.

Scolozzi, P., Jacquier, P., \& Courvoisier, D. S. (2017). Can clinical findings predict orbital fractures and treatment decisions in patients with orbital trauma? Derivation of a simple clinical model. Journal of Craniofacial Surgery, 28(7), e661-e667. 
Research, Society and Development, v. 10, n. 6, e42710615789, 2021

(CC BY 4.0) | ISSN 2525-3409 | DOI: http://dx.doi.org/10.33448/rsd-v10i6.15789

Shah, H. A., Shipchandler, T., Vernon, D., Baumanis, M., Chan, D., Nunery, W. R., \& Lee, H. B. H. (2018). Extra-ocular movement restriction and diplopia following orbital fracture repair. American journal of otolaryngology, 39(1), 34-36.

Snäll, J., Narjus-Sterba, M., Toivari, M., Wilkman, T., \& Thorén, H. (2019). Does postoperative orbital volume predict postoperative globe malposition after blow-out fracture reconstruction? A 6-month clinical follow-up study. Oral and maxillofacial surgery, 23(1), 27-34.

Wi, J. M., Sung, K. H., \& Chi, M. (2017). 'Orbital volume restoration rate after orbital fracture'; a CT-based orbital volume measurement for evaluation of orbital wall reconstructive effect. Eye, 31(5), 713-719.

Young, S. M., Kim, Y. D., Kim, S. W., Jo, H. B., Lang, S. S., Cho, K., \& Woo, K. I. (2018). Conservatively treated orbital blowout fractures: spontaneous radiologic improvement. Ophthalmology, 125(6), 938-944. 